\title{
Surgical approaches to adenocarcinoma of the gastroesophageal junction: the Siewert II conundrum.
}

\author{
Andrew M. Brown \\ Thomas Jefferson University \\ Danica N. Giugliano \\ Thomas Jefferson University \\ Adam C. Berger \\ Thomas Jefferson University \\ Michael J. Pucci \\ Thomas Jefferson University \\ Francesco Palazzo \\ Thomas Jefferson University \\ Follow this and additional works at: https://jdc.jefferson.edu/surgeryfp \\ Part of the Surgery Commons \\ Let us know how access to this document benefits you
}

\section{Recommended Citation}

Brown, Andrew M.; Giugliano, Danica N.; Berger, Adam C.; Pucci, Michael J.; and Palazzo, Francesco, "Surgical approaches to adenocarcinoma of the gastroesophageal junction: the Siewert II conundrum." (2017). Department of Surgery Faculty Papers. Paper 172.

https://jdc.jefferson.edu/surgeryfp/172

This Article is brought to you for free and open access by the Jefferson Digital Commons. The Jefferson Digital Commons is a service of Thomas Jefferson University's Center for Teaching and Learning (CTL). The Commons is a showcase for Jefferson books and journals, peer-reviewed scholarly publications, unique historical collections from the University archives, and teaching tools. The Jefferson Digital Commons allows researchers and interested readers anywhere in the world to learn about and keep up to date with Jefferson scholarship. This article has been accepted for inclusion in Department of Surgery Faculty Papers by an authorized administrator of the Jefferson Digital Commons. For more information, please contact: JeffersonDigitalCommons@jefferson.edu. 
Prepared for: Langenbeck's Archives of Surgery

July 2017 Surgical approaches to adenocarcinoma of the gastroesophageal junction: the
Siewert II conundrum

\author{
Andrew M Brown M.D. \\ Danica Giugliano M.D. \\ Adam C Berger M.D. F.A.C.S. \\ Michael J Pucci M.D. F.A.C.S. \\ Francesco Palazzo M.D. F.A.C.S.
}

Department of Surgery, Thomas Jefferson University Hospital, Philadelphia, PA, USA.

Running Head: Adenocarcinomas of gastroesophageal junction

Author Correspondence:

Francesco Palazzo M.D. F.A.C.S

Associate Professor of Surgery

Department of Surgery

Thomas Jefferson University Hospital

1100 Walnut St. $5^{\text {th }}$ Floor

Philadelphia, PA 19107, USA

Telephone: (215) 955-1622

Fax: (215) 923-8222

Email: Francesco.Palazzo@jefferson.edu 


\section{Abstract \\ Background}

The Siewert classification system for gastroesophageal junction adenocarcinoma has provided morphological and topographical information to help guide surgical decision making. Evidence has shown that Siewert I and III tumors are distinct entities with differing epidemiologic and histologic characteristics and distinct patterns of disease progression, requiring different treatment. Siewert II tumors share some of the characteristics of types I and III lesions, and the surgical approach is not universally agreed upon. Appropriate surgical options include transthoracic esophagogastrectomy, transhiatal esophagectomy, and transabdominal extended total gastrectomy.

\section{Purpose}

A review of the available evidence of the surgical management of Siewert II tumors is presented.

\section{Conclusions}

Careful review of the data appear to support the fact that a satisfactory oncologic resection can be achieved via a transabdominal extended total gastrectomy with a slight advantage in terms of perioperative complications, and overall postoperative quality of life. Overall and disease free survival compares favorably to the transthoracic approach. These results can be achieved with careful selection of patients balancing more than just the Siewert type in the decision making but considering also: preoperative $\mathrm{T}$ and $\mathrm{N}$ stage, histological type (diffuse type requiring longer margins that are not always achievable via gastrectomy), and presence of Barrett's esophagus. 


\section{Key Words}

Gastroesophageal junction adenocarcinoma; Siewert Classification; Esophagectomy; Gastrectomy 


\section{Introduction}

While there has been a decline in the incidence of adenocarcinoma of the distal stomach, adenocarcinoma of the distal esophagus, gastroesophageal (GE) junction, and gastric cardia have increased by $4-10 \%$ per year among males in the United States since $1976[1,2]$. The rise of adenocarcinoma of the distal esophagus and stomach in the United States strongly contrasts to parts of East Asia, where gastric cancer is endemic, and lower esophageal cancer rates continue to grow [3]. These epidemiological changes have been linked to several factors that include the rise of obesity worldwide, as well as increasing rates of gastroesophageal reflux and Barrett's esophagus [4,5]. Obesity has been implicated as the inciting risk factor in almost $40 \%$ of esophageal adenocarcinoma and more than $20 \%$ of gastric cardia adenocarcinoma worldwide [6].

In an attempt to improve the diagnosis and guide the treatment of these tumors, J.R. Siewert developed a topographic-anatomic subclassification of adenocarcinoma of the GE junction as a result of seminal research from the 1980s and 1990s [7,8,9]. Specifically, Siewert and Stein defined adenocarcinoma of the GE junction as tumors with their centers within 5 centimeters proximal or distal to the anatomic cardia. It is critically important to understand that the anatomic landmark in this classification is defined endoscopically, as the oral end of the gastric folds, and not at the Z-line. These tumors were further divided into three types: type I (arising between 1 and $5 \mathrm{~cm}$ proximal to the GE junction) usually develop from an area with specialized intestinal metaplasia of the esophagus (Barrett's esophagus); type II (located within $1 \mathrm{~cm}$ proximal and $2 \mathrm{~cm}$ distal to the gastric cardia) are true adenocarcinoma of the cardia arising from the cardiac epithelium or short segments with intestinal metaplasia at the GE junction, and type III tumors (arising more than $2 \mathrm{~cm}$ 
distal to the GE junction) are subcardial gastric adenocarcinoma which infiltrate the GE junction and distal esophagus from below [10]. This classification system is further illustrated in Figure 1 [11].

The management of these tumors has evolved from surgical resection alone to more recently including adjuvant and neoadjuvant therapies for locally advanced disease. [12]. The ability of preoperative chemoradiation to increase the rates of $\mathrm{R} 0$ resection, as well as positively impact survival has been demonstrated in the CROSS trial. This trial compared neoadjuvant chemoradiotherapy plus surgery was compared to surgery alone for resectable esophageal and GE junctional cancer [13]. A recent Cochrane review suggested that perioperative chemotherapy for resectable gastroesophageal adenocarcinoma increased survival compared to surgical resection alone [14]. Surgical resection remains the backbone of the treatment for GE junction tumors, and tumor location has traditionally guided which operative procedure to utilize.

Based on retrospective review of their large experience, Siewert and his group concluded that type I tumors should be treated with an esophagectomy, while type II and III tumors should be managed with an extended total gastrectomy [15]. This position has come into question in recent years with many groups treating type II tumors more similarly to esophageal adenocarcinoma [11]. It is very telling to note that the $8^{\text {th }}$ edition of the TNM classification classifies Siewert III with gastric cancer, whereas it maintains Siewert I and II as being staged with esophageal cancer [16]. A recent international survey among members of the International Society for Diseases of the Esophagus, the World Organization for Specialized Studies on Disease of the Esophagus, and the International Gastric Cancer Association showed that there are discrepancies in the surgical 
management of these tumors even among experts in the field. While this study was internationally based, it did include a preponderance of European surgeons. 93\% of surgeons preferred esophagectomy for Siewert I tumors, while an extended gastrectomy was favored in $66 \%$ of cases for Siewert II tumors. For type III tumors, gastrectomy was preferred by $90 \%$ of the surgeons [17].

This article reviews the current available evidence regarding type II adenocarcinoma of the gastric cardia with a focus on identifying those factors that may guide the clinician to opt for the surgical technique that may provide the best perioperative outcomes, oncologic results, and quality of life.

\section{Siewert Type II Adenocarcinoma}

Most of the controversy surrounding the management of GE junction adenocarcinomas stems from the fact that these tumors share epidemiologic, histologic, and anatomic characteristics with both esophageal and gastric cancers. Type II tumors show a predilection for males, with a 4.8:1 male to female ratio, and rates of associated intestinal metaplasia (Barrett's) are around 10\% [8]. As compared to type I tumors, where lymph node involvement in the mediastinum reached 50\%, type II tumors, mediastinal nodes were involved in only $11 \%$ of patients in one large series [15]. Further characteristics of these tumors based on Siewert classification are summarized in Table 1 $[10,18]$.

We know that despite advances in neoadjuvant and adjuvant treatments, the backbone of multimodality therapy is an adequate $\mathrm{R} 0$ resection with adequate $\mathrm{LN}$ sampling. Surgical options to be considered for type II tumors include transthoracic 
esophagectomy, transhiatal esophagectomy, a left thoracoabdominal esophagogastrectomy, or a total gastrectomy with extended distal esophageal resection. An assessment of superiority for these techniques must include: oncologic efficacy, perioperative outcomes, overall and disease-free survival, as well as quality of life. Once favored by some groups, proximal gastrectomy is not presently considered among accepted options for treatment due concerns related to oncologic outcomes and poor postoperative quality of life.

Of note, the left thoracoabdominal esophagogastrectomy has been compared to transabdominal total gastrectomy in a randomized controlled trial from the Japan Clinical Oncology Group (ten-year follow-up data have become recently available). The study was stopped after the first interim analysis due to inferiority of the left thoracoabdominal resection in terms of safety and the group recommended against the use of this approach for carcinoma of the gastric cardia [19].

\section{Oncologic Efficacy}

The keys to an oncologically successful surgical resection include obtaining an R0 resection as well as an adequate lymph node harvest.

\section{Resection Margins}

One challenge in obtaining an $\mathrm{R} 0$ resection with tumors at the gastric cardia is the ability to obtain an adequate proximal margin on the esophagus. Classic teaching advocates for a proximal negative margin of a minimum of $5 \mathrm{~cm}$.

Barbour et al studied whether the length of the proximal resection margin and/or the operative approach utilized, had any impact on survival by comparing patients who 
underwent either esophagectomy or extended gastrectomy for Siewert II carcinoma. The authors identified an ex vivo margin length of 3.8 centimeters (that translates to approximately a $5 \mathrm{~cm}$ in vivo margin) correlated with improved survival. When comparing operative approach, they noted median resection margins of $2.0 \mathrm{~cm}$ in the gastrectomy group compared to $5.5 \mathrm{~cm}$ the esophagectomy group ( $\mathrm{p}<0.0001$ ), with overall survival being slightly favored in the esophagectomy group [20]. It is worth noting that the gastrectomy cohort was statistically significantly older, presented with a higher pathologic stage, and had significantly more positive lymph nodes.

A subsequent study from Mine et al reporting on extended gastrectomy for treating Siewert type II (66\%) and III (34\%) demonstrated a very low R1 resection rate (1.4\%) and improved survival with a proximal resection margin of $2.0 \mathrm{~cm}$ ex vivo, and $3.0 \mathrm{~cm}$ in vivo [21]. These authors were careful to consider for gastrectomy only patients that had less than $3 \mathrm{~cm}$ of esophageal involvement on preoperative imaging.

The most recent evidence on the relevance of a proximal margin $>5 \mathrm{~cm}$ comes from the US Gastric Cancer Collaborative. In a report that describes data accumulated from 7 centers in the US, including 162 patients from 2000 to 2012 (Siewert II and III were included - cancers of the cardia or GE junction comprised $67.6 \%$ of the population), the authors found that proximal margin length was not associated with local recurrence or overall survival. They concluded that efforts to achieve a specific proximal margin, especially if it necessitated an esophagectomy, should be abandoned. [22]

These studies indicate that a shorter proximal resection length may prove to be an adequate oncologic margin. Evidence from the literature suggests that the issue of margins cannot be resolved with absolute measures but has to be based on several other factors 
including: histological type, with diffuse type requiring longer margins, presence of Barrett's esophagus, T stage, lymph node metastases, and E-cadherin expression, with negative expression requiring longer margins $[21,23]$.

\section{Lymph Node Metastases}

The argument of whether a thoracic approach should be used for Siewert type II cancers has, at times, been based on the potential risk of leaving positive nodes behind via a transabdominal approach, and thus "under-staging" a patient. One of the earlier descriptions of the pattern of lymph node metastasis for these tumors was provided by Siewert's group examining a case series of over 1500 patients. In patients with type I tumors with $\mathrm{N}+$ disease, $50 \%$ had lower mediastinal nodal metastases, and $15 \%$ had upper mediastinal nodal metastases. By contrast, in type II tumors, lower mediastinal nodal involvement was implicated in only $12 \%$ of lymph node metastases. Only $6 \%$ of type III tumors that metastasized had subsequently spread to the mediastinum [24]. This is further highlighted in Figure 2.

Not surprisingly, the distance from the tumor to the GE junction seems to be an indicator of metastasis to the mediastinal lymph nodes. In a recent study by Kurokawa et al, rates of metastasis or recurrence were significantly higher when the distance from the GE junction to the proximal edge of the tumor was greater than 3 centimeters for upper and middle mediastinal nodes, and greater than 2 centimeters for the lower mediastinal nodes [25]. A more recent report from the same group, looking specifically at clinicopathologic characteristics and prognostic factors of type II tumors found that rates of 
metastases to the lower mediastinal lymph nodes was relatively high (16.1\%), as were metastases to the para-aortic lymph nodes (16.1\%) [26].

A systematic review regarding lymph node metastases in GE junction adenocarcinomas recently confirmed that the most commonly involved lymphatic stations are locoregional (subdiapragmatic, along the left gastric, splenic, and hepatic vessels), and that survival was improved when lymph nodes metastases remained in the locoregional nodes. Rates of metastasis to the lower mediastinal nodal stations ranged from $7.5 \%$ to 23.8\%, while upper mediastinal nodal stations were involved in less than $4 \%$ [27]. These authors pointed out the relevance of the total number of lymph nodes harvested, over specific stations, as being linked to overall survival. The 5-year follow up data from the Dutch trial - that compared transthoracic and transhiatal esophagectomy for Siewert type I and II - confirmed unequivocally that there is no benefit to be derived from an extended lymphadenectomy for type II disease [28].

While many questions, remain and some conflicting reports may be the result of different biology of disease between the eastern and western countries, some conclusions can be made. Taken together, these data suggest that type II tumors preferentially metastasize to the intraabdominal lymph nodes, but the lower mediastinal compartment should be routinely sampled during the dissection of Siewert type II tumors. However, when they do metastasize to mediastinal nodes, this is a predictor of poorer outcomes, and the location relative to the primary tumor is paramount. The distance of the proximal margin of the cancer from the GE junction appears to be a relevant factor in the decision to pursue a transthoracic or a transabdominal approach. 


\section{Perioperative Outcomes}

Siewert and his colleagues compared short-term morbidity and mortality of extended gastrectomy versus transhiatal esophagectomy for type II tumors. The authors found a $1.9 \%$ mortality in the extended gastrectomy group compared to $5.6 \%$ in the esophagectomy group. Despite the differences in mortality favoring extended gastrectomy, following multivariate analysis, the single most important prognostic factor impacting survival, was attaining an R0 resection [29].

A recent database analysis from the University of Kentucky using the American College of Surgeons National Surgical Quality Improvement Project (ACS-NSQIP) and the Surveillance Epidemiology and End Results (SEER) databases looked at a head-to-head comparison of esophagectomy and total gastrectomy for type II tumors. When examining 1181 patients from the NSQIP database, the authors found no significant differences in length of stay (10.5 days in gastrectomy, 10.0 in esophagectomy, $\mathrm{p}=0.46)$, morbidity (33.2\% in gastrectomy, $35 \%$ in esophagectomy, $\mathrm{p}=0.62$ ), and thirty-day mortality ( $3.7 \%$ in gastrectomy, $2.4 \%$ in esophagectomy, $\mathrm{p}=0.26$ ) [30].

\section{Long-term Outcomes}

The results of the Dutch trial have been briefly mentioned before. Omloo et al reported on a five-year follow-up of a randomized clinical trial comparing transthoracic and transhiatal esophagectomy for types I and II tumors. In a subgroup analysis for type II tumors, there was only a negligible difference in overall five-year survival-31\% following transhiatal esophagectomy and $27 \%$ following transthoracic esophagectomy $(\mathrm{p}=0.81)$ [28]. 
Long-term oncologic outcomes were also examined by Martin et al. in their SEER database analysis. Unadjusted median overall survival favored esophagectomy (26.0 vs. 21.0 months, $p=0.025)$, but with multivariate analysis, surgical approach was not an independent predictor of overall survival. Notably, a higher percentage of patients underwent multimodality therapy in the group that underwent esophagectomy versus gastrectomy ( $42.9 \%$ vs. $29.6 \%, \mathrm{p}<0.001$ ). The authors of this study concluded that multimodality therapy and tumor biology more so than surgical approach impacted the oncologic outcomes for Type II tumors [30].

Qualtiy of Life

Patient-centered outcomes, with a specific interest in post-operative quality of life (QOL), are very important considerations in patients undergoing major gastroesophageal resections. A recent study looked at long-term QOL comparing extended gastrectomy versus transthoracic esophagectomy for GE junction adenocarcinoma. The authors looked at 123 patients with a standardized QOL questionnaire, and then a specific QOL instrument for esophageal cancer specific symptoms. When compared to a reference population of healthy volunteers, predictably both esophagectomy and gastrectomy fared worse in almost every category at two years following surgery. In a head-to-head comparison, extended gastrectomy with D2 lymphadenectomy had statistically significant advantages in physical function ( $\mathrm{p}=0.04)$, dyspnea $(\mathrm{p}=0.02)$, and reflux $(\mathrm{p}=0.03)$. All other surveyed areas provided no significant differences between the two operations [31]. 


\section{Beyond the Siewert Classification}

The Siewert classification has provided the surgical community with a powerful tool to guide decision making for a very challenging subgroup of cancers. It relies heavily on accurate preoperative characterization. Generally staging of adenocarcinomas of the GEJ is accomplished through endoscopic ultrasound (EUS) and computed tomography (CT). These studies yield information regarding tumor location, depth of invasion, and evidence of nodal disease. Unfortunately, neither are particularly accurate in tumor localization. In particular, for Siewert II tumors, EUS localized tumors $66 \%$ of the time, and CT $57 \%$ of the time, as compared to final pathology [32]. Unfortunately, the best techniques currently available frequently may fail in accurately localizing a tumor by Siewert location, and thus inaccurately guide surgical therapy. Data from the Dutch trial demonstrated that there was significant migration of patients from having a type I tumor on preoperative endoscopy to having a type II tumor on final pathology. There were 180 patients classified as type I on preoperative endoscopic assessment but only 90 patients were confirmed type I on final path [28].

Review of the available data presented above, seem to point to the fact that a careful and meticulous preoperative identification of the tumor location with respect to the GE junction, accurate staging and consideration of the biologic features of the cancer (Lauren classification [33], presence of intestinal metaplasia) are key in determining the best surgical approach to Siewert II disease. Emerging immune-histochemical techniques, including but not limited to the expression of the proto-oncogene Her2 - expressed in a subset of patients - may likely add to the available data and guide the clinician in their decision making [34]. 


\section{Conclusions}

There are evident conflicts in the available literature that are the result of several levels of bias. Surgeons from different parts of the world have radically different philosophical approaches to this disease, possibly the result of only partially known epidemiological differences, and the available data is mixed as it frequently combines Type II and III together. For these reasons, final considerations are difficult to make. Any technique chosen for the surgical resection of type II tumors should have the same goals: achieving a negative margin, performing an adequate lymphadenectomy, limiting the perioperative morbidity and mortality of the operation, and attaining optimal patientcentered quality of life. Based on the above review of the evidence, there appears to be a slight advantage to undergoing extended gastrectomy over esophagectomy for Siewert type II adenocarcinomas in the right subgroup of patients, when an oncologically radical procedure can be completed. The choice of surgical technique should not be based solely on anatomic location, but should rely on careful evaluation by a multidisciplinary team while considering endoscopic features, staging results, and histologic features. 


\section{Compliance with Ethical Standards}

Conflict of Interest: The authors declare that they have no conflict of interest.

Ethical approval: This article does not contain any studies with human participants or animals performed by any of the authors.

Informed consent: This was a review article, but to our knowledge, for all studies that were included: informed consent was obtained from all individual participants included in the study. 


\section{References}

1. Crew KD, Neugut AI. Epidemiology of upper gastrointestinal malignancies. Semin Oncol. 2004;31(4):450-464.

2. Devesa SS, Fraumeni JF. The rising incidence of gastric cardia cancer. J Natl Cancer Inst. 1999;91(9):747-749.

3. Ferlay J, Shin HR, Bray F, Forman D, Mathers C, Parkin DM. Estimates of worldwide burden of cancer in 2008: GLOBOCAN 2008. Int J Cancer. 2010;127(12):2893-2917.

4. Holmes RS, Vaughan TL. Epidemiology and pathogenesis of esophageal cancer. Semin Radiat Oncol. 2007;17(1):2-9.

5. Turati F, Tramacere I, La Vecchia C, Negri E. A meta-analysis of body mass index and esophageal and gastric cardia adenocarcinoma. Ann Oncol. 2013;24(3):609-617.

6. Olsen CM, Pandeya N, Green AC, Webb PM, Whiteman DC, Study AC. Population attributable fractions of adenocarcinoma of the esophagus and gastroesophageal junction. Am J Epidemiol. 2011;174(5):582-590.

7. Siewert JR, Hölscher AH, Becker K, Gössner W. [Cardia cancer: attempt at a therapeutically relevant classification]. Chirurg. 1987;58(1):25-32.

8. Siewert JR, Feith M, Stein HJ. Biologic and clinical variations of adenocarcinoma at the esophago-gastric junction: relevance of a topographic-anatomic subclassification. J Surg Oncol. 2005;90(3):139-146; discussion 146.

9. Gertler R, Stein HJ, Loos M, Langer R, Friess H, Feith M. How to classify adenocarcinomas of the esophagogastric junction: as esophageal or gastric cancer? Am J Surg Pathol. 2011;35(10):1512-1522. 
10. Siewert JR, Stein HJ. Classification of adenocarcinoma of the oesophagogastric junction. BrJ Surg. 1998;85(11):1457-1459.

11. Mariette C, Piessen G, Briez N, Gronnier C, Triboulet JP. Oesophagogastric junction adenocarcinoma: which therapeutic approach? Lancet Oncol. 2011;12(3):296-305.

12. D'Journo XB, Thomas PA. Current management of esophageal cancer.J Thorac Dis. 2014;6 Suppl 2:S253-264.

13. Shapiro J, van Lanschot JJ, Hulshof MC, van Hagen P et al. Neoadjuvant chemoradiotherapy plus surgery versus surgery alone for oesophageal or junctional cancer (CROSS): long-term results of a randomized controlled trial. Lancet Oncol. 2015;16(9):1090-1098.

14. Ronellenfitsch U, Schwarzbach M, Hofheinz R, et al. Perioperative chemo(radio)therapy versus primary surgery for resectable adenocarcinoma of the stomach, gastroesophageal junction, and lower esophagus. Cochrane Database Syst Rev. 2013(5):CD008107.

15. Feith M, Stein HJ, Siewert JR. Adenocarcinoma of the esophagogastric junction: surgical therapy based on 1602 consecutive resected patients. Surg Oncol Clin N Am. 2006;15(4):751-764.

16. Brierley J, Gospodarowicz MK, Wittekind C. TNM classification of malignant tumours. Eighth edition. ed. Chichester, West Sussex, UK ; Hoboken, NJ: John Wiley \& Sons, Inc.; 2017.

17. Haverkamp L, Seesing MF, Ruurda JP, Boone J et al. Worldwide trends in surgical techniques in the treatment of esophageal and gastroesophageal junction cancer. Dis Esophagus. 2017;30(1):1-7. 
18. Kurokawa Y, Sasako M, Doki Y. Treatment Approaches to Esophagogastric Junction Tumors. Dig Surg. 2013;30:169-173.

19. Kurokawa Y, Sasko M, Sano T, Yoshikawa T et al. Ten-year follow-up results of a randomized clinical trial comparing left thoracoabdominal and abdominal transhiatal approaches to total gastrectomy for adenocarcinoma of the oesophagogastric junction or gastric cardia. Br J Surg. 2015;102(4)341-348.

20. Barbour AP, Rizk NP, Gonen M, et al. Lymphadenectomy for adenocarcinoma of the gastroesophageal junction (GEJ): impact of adequate staging on outcome. Ann Surg Oncol. $2007 ; 14(2): 306-316$.

21. Mine S, Sano T, Hiki N, et al. Proximal margin length with transhiatal gastrectomy for Siewert type II and III adenocarcinomas of the oesophagogastric junction. Br J Surg. 2013;100(8):1050-1054.

22. Postlewait LM, Squires MH $3^{\text {rd }}$, Kooby DA, Poultsides GA et al. The importance of the proximal resection margin distance for proximal gastric adenocarcinoma: A multiinstitutional study of the US Gastric Cancer Collaborative. J Surg Oncol. 2015;112:203207.

23. Pedrazzani C. Should adenocarcinoma of the esophagogastric junction be classified as gastric or esophageal cancer, or else as a distinct clinical entity? Ann Surg. 2015;261(4):e107-108.

24. Feith M, Stein HJ, Siewert JR. Adenocarcinoma of the Esophagogastric Junction: Surgical Therapy Based on 1602 Consecutive Resected Patients. Surg Oncol Clin N Am. 2006;15:751-764. 
25. Kurokawa Y, Hiki N, Yoshikawa T, Kishi K et al. Mediastinal lymph node metastasis and recurrence in adenocarcinoma of the esophagogastric junction. Surgery. 2015;157(3):551-555.

26. Matsuda T, Kurokawa Y, Yoshikawa T, Kishi K et al. Clinicopathological Characteristics and Prognostic Factors of Patients with Siewert Type II Esophagogastric Junction Carcinoma: A Retrospective Multicenter Study. World J Surg. 2016;40(7):1672-1679.

27. Okholm C, Svendsen LB, Achiam MP. Status and prognosis of lymph node metastasis in patients with cardia cancer - a systematic review. Surg Oncol. 2014;23(3):140-146.

28. Omloo JM, Lagarde SM, Hulscher JB, et al. Extended transthoracic resection compared with limited transhiatal resection for adenocarcinoma of the mid/distal esophagus: five-year survival of a randomized clinical trial. Ann Surg. 2007;246(6):992-1000; discussion 1000-1001.

29. von Rahden BH, Stein HJ, Siewert JR. Surgical management of esophagogastric junction tumors. World J Gastroenterol. 2006;12(41):6608-6613.

30. Martin JT, Mahan A, Zwischenberger JB, McGrath PC, Tzeng CW. Should gastric cardia cancers be treated with esophagectomy or total gastrectomy? A comprehensive analysis of 4,996 NSQIP/SEER patients. J Am Coll Surg. 2015;220(4):510-520.

31. Fuchs H, Hölscher AH, Leers J, et al. Long-term quality of life after surgery for adenocarcinoma of the esophagogastric junction: extended gastrectomy or transthoracic esophagectomy? Gastric Cancer. 2016;19(1):312-317.

32. Parry K, Haverkamp L, Bruijnen RC, Siersema PD et al. Staging of adenocarcinoma of the gastroesophageal junction. Eur J Sur Oncol. 2016;42(3):400-406. 
33. Lee JH, Chang KK, Yoon C, Tang LH et al. Lauren Histologic Type Is the Most Important Factor Associated With Pattern of Recurrence Following Resection of Gastric Adenocarcinoma. Ann Surg. 2016 [Epub ahead of print].

34. Bartley AN, Washington MK, Colasacco C, Ventura CB et al. HER2 Testing and Clinical Decision Making in Gastroesophageal Adenocarcinoma: Guidelines From the College of American Pathologists, American Society for Clinical Pathology, and the American Society of Clinical Oncology. J Clin Oncol. 2017;35(4):446-464. 